\title{
The effects of local infiltration anesthesia and femoral nerve block analgesia after total knee arthroplasty: a systematic review and meta-analysis
}

\author{
Qiang Liu $^{1 \#}$, Anli Wang' ${ }^{2}$ Jixia Zhang ${ }^{2 \#}$ \\ ${ }^{1}$ Department of International Painless Medical Center, Tianjin Stomatological Hospital, School of Medicine, Nankai University, Tianjin, China; \\ ${ }^{2}$ Department of Pediatric Dentistry, Tianjin Stomatological Hospital, School of Medicine, Nankai University, Tianjin, China \\ Contributions: (I) Conception and design: Q Liu; (II) Administrative support: J Zhang; (III) Provision of study materials or patients: A Wang; (IV) \\ Collection and assembly of data: Q Liu; (V) Data analysis and interpretation: J Zhang; (VI) Manuscript writing: All authors; (VII) Final approval of \\ manuscript: All authors. \\ "These authors contributed equally to this work and should be considered as co-first authors. \\ Correspondence to: Jixia Zhang. Department of Pediatric Dentistry, Tianjin Stomatological Hospital, School of Medicine, Nankai University, Tianjin \\ 300041, China. Email: zhangjixia415@126.com.
}

Background: Local infiltration anesthesia (LIA) and femoral nerve block (FNB) are commonly used analgesia methods after total knee arthroplasty (TKA). However, there is no definitive conclusion about which of these two analgesia modes is superior. Therefore, this study aimed to systematically evaluate the analgesic effects of LIA and FNB after TKA.

Methods: We used the terms "total knee replacement, knee replacement, total knee arthroplasty, knee arthroplasty, local infiltration analgesia, periarticular infiltration, periarticular injection, intra-articular infiltration, intra-articular injection, peripheral nerve block, femoral nerve block" to search the PubMed, Cochrane Central Register of Controlled Trials, Embase, Web of Science, China National Knowledge Infrastructure (CNKI), Wanfang, and Weipu databases. The search period was set from the date of establishment of the database to September 2021. The Cochrane risk of bias tool was used to evaluate the quality of the included studies, and network meta-analysis was performed using Stata14.0 and RevMan 5.30 software.

Results: Nine articles were included for analysis. The results of meta-analysis showed that compared with LIA and FNB, the difference in opioid use [mean difference (MD) $-4.35,95 \%$ confidence interval (CI): -7.26 to -1.45 ] was statistically significant. However, there was no significant difference between the static visual analogue score at 24 hours postoperatively (MD 0.20, 95\% CI: -0.91 to 1.31 ), the visual analogue score for exercise visual analogy at 24 hours after surgery (MD 0.10, 95\% CI: -0.12 to 0.32 ), and the length of hospital stay (MD 0.05, 95\% CI: -0.40 to 0.50 ).

Discussion: LIA and FNB have similar effects on pain relief after TKA, but LIA can reduce the use of analgesic drugs and is easy to operate. Therefore, LIA can be used as the priority analgesic method for patients with TKA. However, multi-center, large-sample, high-quality, randomized controlled trials are still needed for further verification.

Keywords: Total knee replacement; local infiltration anesthesia (LIA); femoral nerve block (FNB); meta-analysis

Submitted Nov 30, 2021. Accepted for publication Jan 30, 2022.

doi: $10.21037 / \mathrm{atm}-22-286$

View this article at: https://dx.doi.org/10.21037/atm-22-286 


\section{Introduction}

Total knee arthroplasty (TKA) is one of the most effective and widely used treatments for end-stage osteoarthritis and rheumatoid arthritis (1). Although effective pain control after total knee replacement can speed up recovery, shorten hospital stays, and reduce overall costs, the pain after total knee replacement is usually very severe and difficult to relieve (2). Local infiltration anesthesia (LIA) and femoral nerve block (FNB) are common postoperative analgesia methods and have been widely used clinically.

Although FNB is a widely accepted and commonly used post-TKA regional anesthesia technique by doctors, there are reports that some patients still experience significant postoperative pain after receiving $\mathrm{FNB}(3,4)$. Compared with peripheral nerve block, LIA is an alternative and convenient anesthesia technique that is usually performed by surgeons. At the same time, the efficacy and safety of LIA are comparable to epidural anesthesia, FNB, and intrathecal morphine (5). Therefore, anesthesia by LIA is a good option to supplement FNB for pain relief after TKA (6-8). However, as important analgesic methods of TKA, a consensus has not yet been reached as to which of these anesthesia methods, LIA or FNB, is more suitable for pain relief.

Therefore, this article aims to systematically evaluate the analgesic effects of LIA and FNB after TKA, in order to provide a reference for the selection of the best analgesic mode after TKA. We present the following article in accordance with the PRISMA reporting checklist (available at https://atm.amegroups.com/article/view/10.21037/atm$22-286 /$ rc).

\section{Methods}

\section{Search strategy}

We performed a literature search of English biomedical databases (PubMed, Cochrane Central Register of Controlled Trials, Embase, and Web of Science) and major Chinese biomedical databases [China National Knowledge Infrastructure (CNKI), Wanfang, and Weipu]. The following search terms were used: "total knee replacement, knee replacement, total knee arthroplasty, knee arthroplasty, local infiltration analgesia, periarticular infiltration, periarticular injection, intra-articular infiltration, intraarticular injection, peripheral nerve block, femoral nerve block". The retrieval time was from the date of establishment of the database to September 2021.

\section{Inclusion and exclusion criteria}

The inclusion criteria were as follows: (I) research subjects: the patients were adults who received total knee replacement; (II) intervention measures: comparison of two analgesia methods in the LIA and FNB groups; (III) research type: prospective randomized controlled trial; and (IV) the reported outcome indicators included one or more of the following: the amount of opioids used, the static visual analogue score at $24 \mathrm{~h}$ postoperatively, the exercise visual analogue score at $24 \mathrm{~h}$ postoperatively, and the length of hospital stay. The exclusion criteria were as follows: (I) non-primary total knee replacement surgery, unicondylar joint replacement, and knee arthroscopy; (II) the main data is incomplete, and the author has not been contacted to obtain valid data; (III) studies involving minor patients; and (IV) retrospective research.

\section{Article screening and data extraction}

Two reviewers independently read the titles and abstracts of the retrieved articles to determine their eligibility for inclusion in this meta-analysis. If any reviewer believed that an article met the inclusion/exclusion criteria, the full text was reviewed and the data was extracted. Disputes about the qualification of the articles were resolved through discussion. If additional data was required, the reviewers contacted the corresponding authors of the article to obtain it. The patients included in this article were divided into two groups: LIA group and FNB group. The data was also independently extracted by two reviewers based on the preestablished data sheet, including the author's name, country, year of publication, journal name, and number of patients.

\section{Quality assessment}

The Cochrane risk of bias tool was used to assess the quality and risk of bias of the included articles. The evaluation items included the following: random sequence generation, allocation concealment, blinding of investigators and subjects, blinded evaluation of research outcomes, completeness of outcome data, selective outcome reporting, and other biases. According to the evaluation, the biases were classified into high-risk, low-risk, and unclear. Two reviewers carried out the above process separately, and 


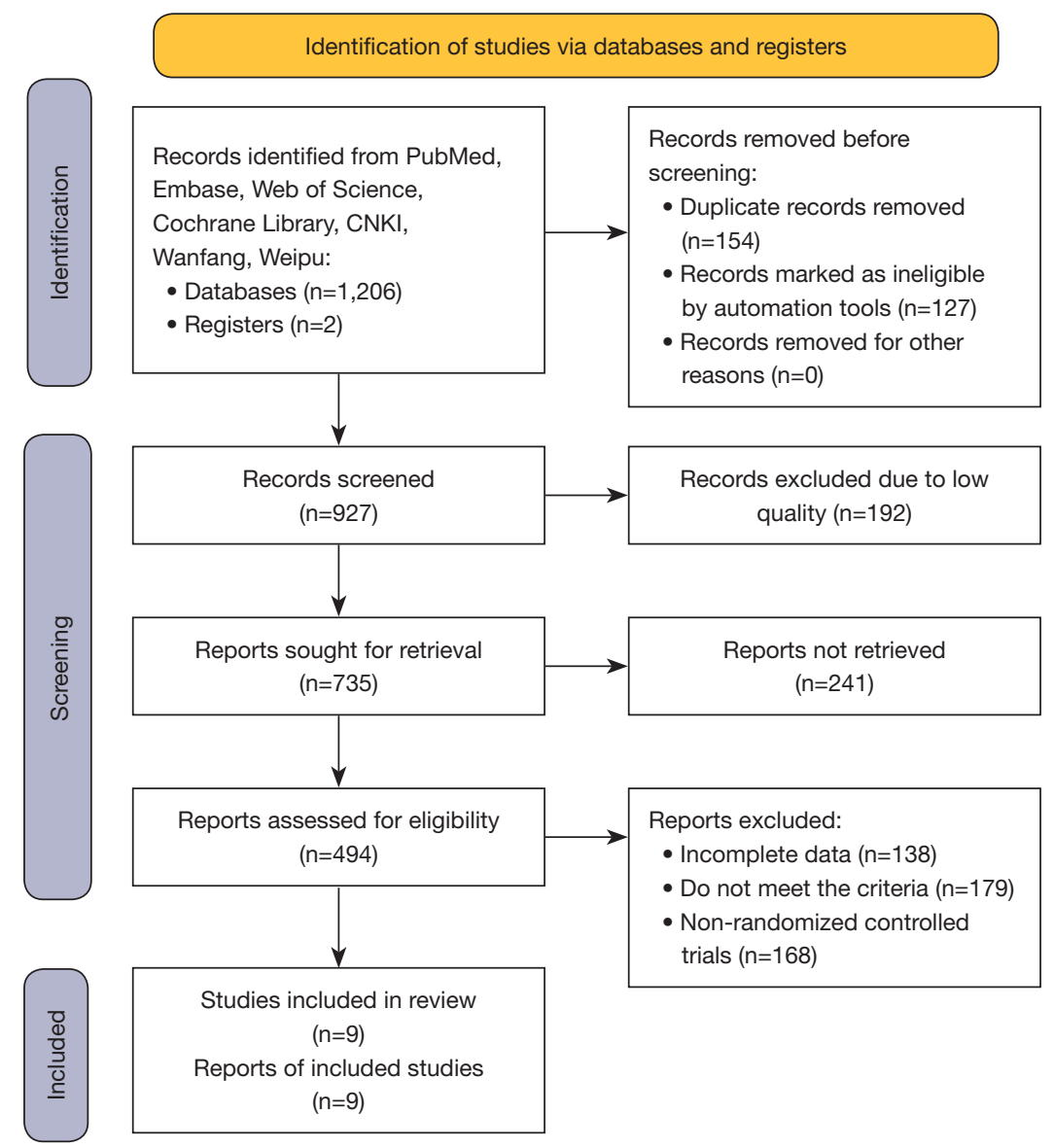

Figure 1 Flow diagram of the search, screening, and inclusion process.

differences were resolved through discussion.

\section{Statistical analysis}

Percentages and relative risk (RR) or mean difference (MD) with $95 \%$ confidence interval (CI) were used to describe the data, and the $\mathrm{I}^{2}$ test was used to test the heterogeneity of the included articles. If the heterogeneity between studies was small $\left(\mathrm{P}>0.1, \mathrm{I}^{2}<50 \%\right)$, the fixed-effects model was used to merge the effect sizes; however, if the heterogeneity between studies was obvious ( $\mathrm{P} \leq 0.1, \mathrm{I}^{2} \geq 50 \%$ ), the random effects model was used to merge the effect size. And the sensitivity analysis was carried out according to the Cochrane systematic review method. Statistical analysis and graphs were performed using Stata14.0 (StataCorp, China) and RevMan 5.30 software provided by Cochrane Collaboration. $\mathrm{P} \leq 0.05$ was considered to indicate a statistically significant difference. A funnel plot was used to check the risk of publication bias.

\section{Results}

\section{Search results and study characteristics}

After the database confirmed the records and eliminated records that did not satisfy the research, 927 records were screened out. 735 records were obtained after excluding 192 low-quality records, and 241 reports not retrieved, finally 494 articles were retrieved. After reading the full texts and excluding articles with incomplete data, non-randomized controlled experiments, and those with no research indicators according to the inclusion and exclusion criteria, nine articles that could be used for meta-analysis were finally obtained. The specific process is shown in Figure 1 . A total of 228 patients with LIA and 269 patients with FNB were included in the nine articles. All of the selected articles had a clear diagnosis and met the inclusion and exclusion 
Table 1 Basic characteristics of the study articles

\begin{tabular}{|c|c|c|c|c|c|}
\hline Author & Country & Year & Journal & LIA group (n) & FNB group $(n)$ \\
\hline Kovalak et al. (10) & Turkey & 2015 & Acta Orthop Traumatol Turc & 30 & 30 \\
\hline Ashraf et al. (11) & UK & 2013 & The Knee & 19 & 21 \\
\hline Chaumeron et al. (12) & France & 2013 & Clin Orthop Relat Res & 29 & 30 \\
\hline Choi et al. (14) & Canada & 2016 & Anesth Analg & 41 & 79 \\
\hline Kurosaka et al. (15) & Japan & 2016 & $J$ Arthroplasty & 21 & 21 \\
\hline Moghtadaei et al. (16) & Iran & 2014 & Iran Red Cres Med J & 18 & 18 \\
\hline Varshney et al. (17) & India & 2019 & Anaesthesia, Pain \& Intensive Care & 30 & 30 \\
\hline
\end{tabular}

LIA, local infiltration anesthesia; FNB, femoral nerve block.

criteria. The remaining basic characteristics of the articles are shown in Table 1.

The quality assessment results showed that in terms of random sequence generation bias, one study was high risk, six studies were low risk, and the risk of the remaining two studies was unclear. For allocation concealment bias, two studies were high-risk, six studies were low-risk, and the risk of the remaining study was unclear. As for the blinding biases of participants and researchers, four studies were high-risk and five studies were low-risk. With regards to the blinded bias of outcome evaluation, three studies were highrisk, five studies were low-risk, and the risk of the remaining study was unclear. All studies had a low risk of incomplete outcome data bias. Regarding selective reporting domain bias, five studies were low risk and four studies had an unclear risk. As for other biases, six studies were low-risk, and three studies had an unclear risk, as shown in Figure 2.

\section{Meta-analysis results}

The amount of opioids used within $\mathbf{2 4} \mathrm{h}$ postoperatively Based on the inclusion and exclusion criteria, a total of seven articles were included for meta-analysis of the differences in the use of opioids within 24 hours after TKA by LIA and FNB anesthesia. The analysis results showed that $\mathrm{P}=0.000$ and $\mathrm{I}^{2}=78.5 \%$, suggesting that there was heterogeneity in the opioid use between the two analgesia methods, so the random effects model was used for combination analysis. The combined effect size $\mathrm{MD}$ was $-4.35,95 \%$ CI: $(-7.26,-1.45)$, as shown in Figure 3. The results of the comprehensive effect size test were $Z=-2.935$ and $\mathrm{P}=0.003$. Therefore, the meta-analysis results indicated that there was a statistically significant difference in the amount of opioids between the LIA and FNB groups at 24 hours after surgery.

\section{Twenty-four hours resting visual analogy score after operation}

Seven articles were included for meta-analysis of the difference between LIA and FNB anesthesia on the resting visual analog score within 24 hours after TKA. The analysis results showed that $\mathrm{P}=0.000$ and $\mathrm{I}^{2}=94.7 \%$, suggesting that there was heterogeneity in the resting visual analog scores between the two analgesia methods, so the random effects model was used for combined analysis. The combined effect size MD was $0.20,95 \%$ CI: $(-0.91,1.31)$, as shown in Figure 4 . The results of the comprehensive effect size test were $\mathrm{Z}=0.359$ and $\mathrm{P}=0.719$. Therefore, the meta-analysis results indicated that there was no statistical difference between the LIA and FNB anesthesia groups in the $24 \mathrm{~h}$ resting visual analog score after surgery.

\section{Twenty-four hours exercise visual analogy score after operation}

Five articles were used for meta-analysis of the difference between LIA and FNB anesthesia on the exercise visual analog scores within 24 hours after TKA. The analysis results showed that $\mathrm{P}=0.888$ and $\mathrm{I}^{2}=0.0 \%$, suggesting that there was homogeneity in the exercise visual analog scores between the two analgesia methods, so the fixed effects model was used for combined analysis. The combined effect size MD was $0.10,95 \%$ CI: $(-0.12,0.32)$, as shown in 


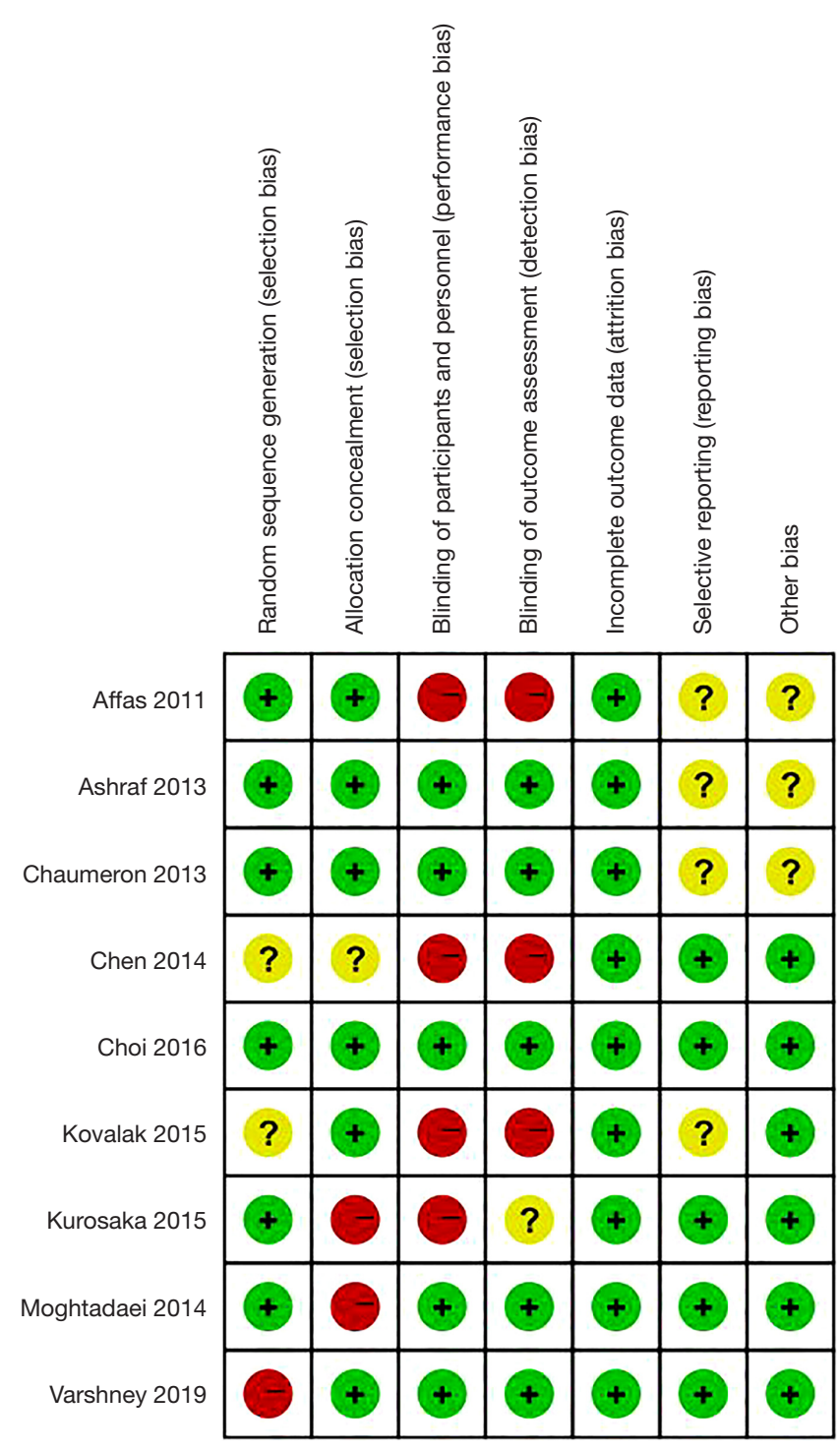

Figure 2 Literature quality evaluation details.

Figure 5. The results of the comprehensive effect size test were $\mathrm{Z}=0.878$ and $\mathrm{P}=0.380$. Therefore, the meta-analysis showed that there was no statistical difference in the exercise visual analog scores of the LIA and FNB anesthesia groups at 24 hours after surgery.

\section{Hospital stay}

Five articles were included for meta-analysis of the difference between LIA and FNB anesthesia on the length of hospital stay after TKA. The analysis results showed that $\mathrm{P}=0.067$ and $\mathrm{I}^{2}=54.3 \%$, suggesting that there was heterogeneity in the length of hospital stay between the two analgesia methods, so the random effects model was used for combined analysis. The combined effect size MD was $0.05,95 \%$ CI: $(-0.40,0.50)$, as shown in Figure 6 . The results of the comprehensive effect size test were $Z=0.211$ and $\mathrm{P}=0.833$. Therefore, the meta-analysis results indicated that there was no statistical difference in the postoperative hospital stay between the LIA and FNB anesthesia groups.

\section{Publication bias}

A funnel plot was used to check the publication bias of the amount of opioids used in the $24 \mathrm{~h}$ postoperatively. Analysis of the funnel plots showed asymmetry, indicating that there may be publication bias (Figure 7).

\section{Risk of bias}

Among the eligible studies, the random sequence generation bias assessment showed that one article was high risk (17), six articles were low risk $(9,11,12,14-16)$, and the risk of the remaining two articles was unclear $(10,13)$. The allocation concealment bias assessment showed that two articles were high risk $(15,16)$, six articles were low risk $(9-12,14,17)$, and the risk of the remaining article was unclear (13). For the blinding biases of participants and researchers, four articles were high risk $(9,10,13,15)$ and five articles were low risk $(11,12,14,16,17)$. The outcome assessment blinding bias analysis showed that three articles were high risk $(9,10,13)$, five articles were low risk $(11,12,14,16,17)$, and the risk of the remaining article was unclear (15). All studies had a low risk of bias for incomplete outcome data. Regarding selective reporting domain bias, five articles were low risk (13-17) and four articles were unclear (9-12). As for other biases, six articles were low risk $(10,13-17)$ and three articles had an unclear risk $(9,11,12)$, as shown in Figure 8.

\section{Discussion}

Pain is the most common complaint after TKA. Patients often suffer from poor postoperative analgesia, resulting in poor knee rehabilitation, slow functional recovery, and prolonged hospital stay. This reduces the effectiveness of treatment and also increases the economic burden of patients (18). FNB is an injection of anesthetic around the femoral nerve to achieve the purpose of pain relief, is currently considered to be the best option for analgesia after TKA $(19,20)$, but continuous FNB has catheter loss, catheter-related infection, and weakened quadriceps muscle strength, which affect the postoperative functional activities of patients and increases their risk of falls (1.6-2.7\%) and 


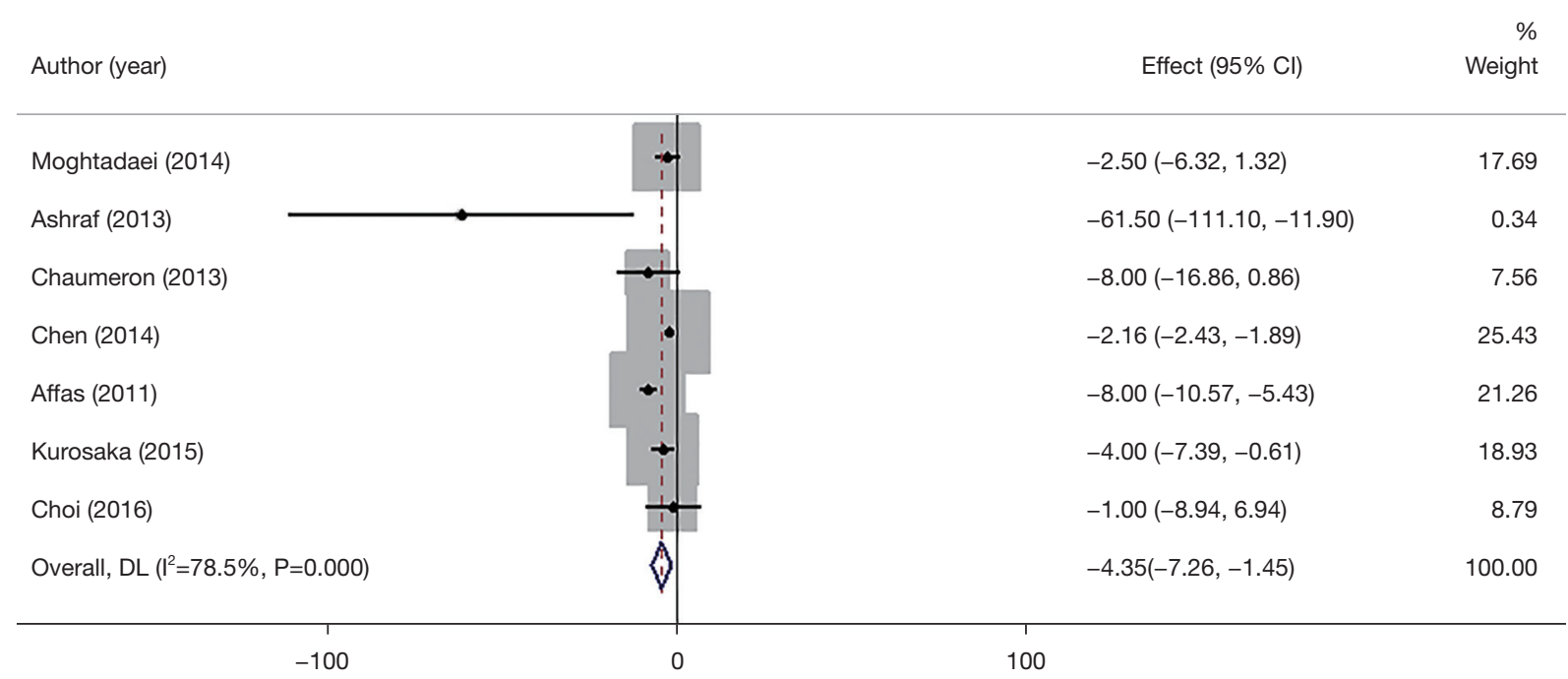

NOTE: Weights are from random-effects model

Figure 3 Forest plot of effectiveness of the amount of opioids used within 24 h postoperatively. Comparison of the amount of opioids used within $24 \mathrm{~h}$ postoperatively between the LIA and FNB anesthesia groups. Statistical method: inverse variance of random effects model (MD and 95\% CI). LIA, local infiltration anesthesia; FNB, femoral nerve block; MD, mean difference; CI, confidence interval.

\begin{tabular}{|c|c|c|c|}
\hline Author (year) & & Effect $(95 \% \mathrm{Cl})$ & $\begin{array}{r}\% \\
\text { Weight }\end{array}$ \\
\hline Varshney (2019) & $\longrightarrow$ & $2.27(1.93,2.61)$ & 15.39 \\
\hline Ashraf (2013) & 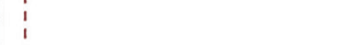 & $-1.50(-2.93,-0.07)$ & 12.41 \\
\hline Chaumeron (2013) & +1 & $0.00(-0.51,0.51)$ & 15.12 \\
\hline Kovalak (2015) & 一 & $1.26(0.56,1.96)$ & 14.72 \\
\hline Affas (2011) & 1 & $0.00(-1.02,1.02)$ & 13.79 \\
\hline Kurosaka (2015) & 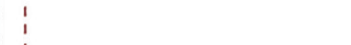 & $-0.80(-1.50,-0.10)$ & 14.71 \\
\hline Choi (2016) & & $-0.20(-1.20,0.80)$ & 13.86 \\
\hline Overall, $\mathrm{DL}\left(\mathrm{I}^{2}=94.7 \%, \mathrm{P}=0.000\right)$ & 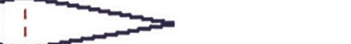 & $0.20(-0.91,1.31)$ & 100.00 \\
\hline
\end{tabular}

NOTE: Weights are from random-effects model

Figure 4 Forest plot of $24 \mathrm{~h}$ rest visual analogy score after surgery. Comparison of $24 \mathrm{~h}$ rest visual analogy score postoperatively between the LIA and FNB anesthesia groups. Statistical method: inverse variance of the random effects model (MD and 95\% CI). LIA, local infiltration anesthesia; FNB, femoral nerve block; MD, mean difference; CI, confidence interval.

other issues, and some patients even require reoperation $(0.4 \%)(21)$. Since the innervation of the knee joint comes from the femoral nerve, sciatic nerve and obturator nerve, etc., blocking the femoral nerve alone cannot completely block the conduction of pain. There are still some patients who feel pain and discomfort in the popliteal area behind the knee joint. To a certain extent, it also affects the patient's postoperative rehabilitation training. 


\begin{tabular}{|c|c|c|}
\hline Author (year) & Effect $(95 \% \mathrm{Cl})$ & $\begin{array}{r}\% \\
\text { Weight }\end{array}$ \\
\hline Chen (2014) & $0.10(-0.15,0.35)$ & 80.01 \\
\hline Chaumeron (2013) & $0.10(-1.23,1.43)$ & 2.79 \\
\hline Kovalak (2015) & $0.15(-0.54,0.84)$ & 10.30 \\
\hline Affas (2011) & $0.50(-0.74,1.74)$ & 3.20 \\
\hline Choi (2016) & $-0.40(-1.55,0.75)$ & 3.70 \\
\hline Overall, IV $\left(I^{2}=0.0 \%, P=0.888\right)$ & $0.10(-0.12,0.32)$ & 100.00 \\
\hline
\end{tabular}

Figure 5 Forest plot of $24 \mathrm{~h}$ exercise visual analogy score after surgery. Comparison of $24 \mathrm{~h}$ exercise visual analogy score postoperatively between the LIA and FNB anesthesia groups. Statistical method: inverse variance of the fixed effects model (MD and 95\% CI). LIA, local infiltration anesthesia; FNB, femoral nerve block; MD, mean difference; CI, confidence interval.

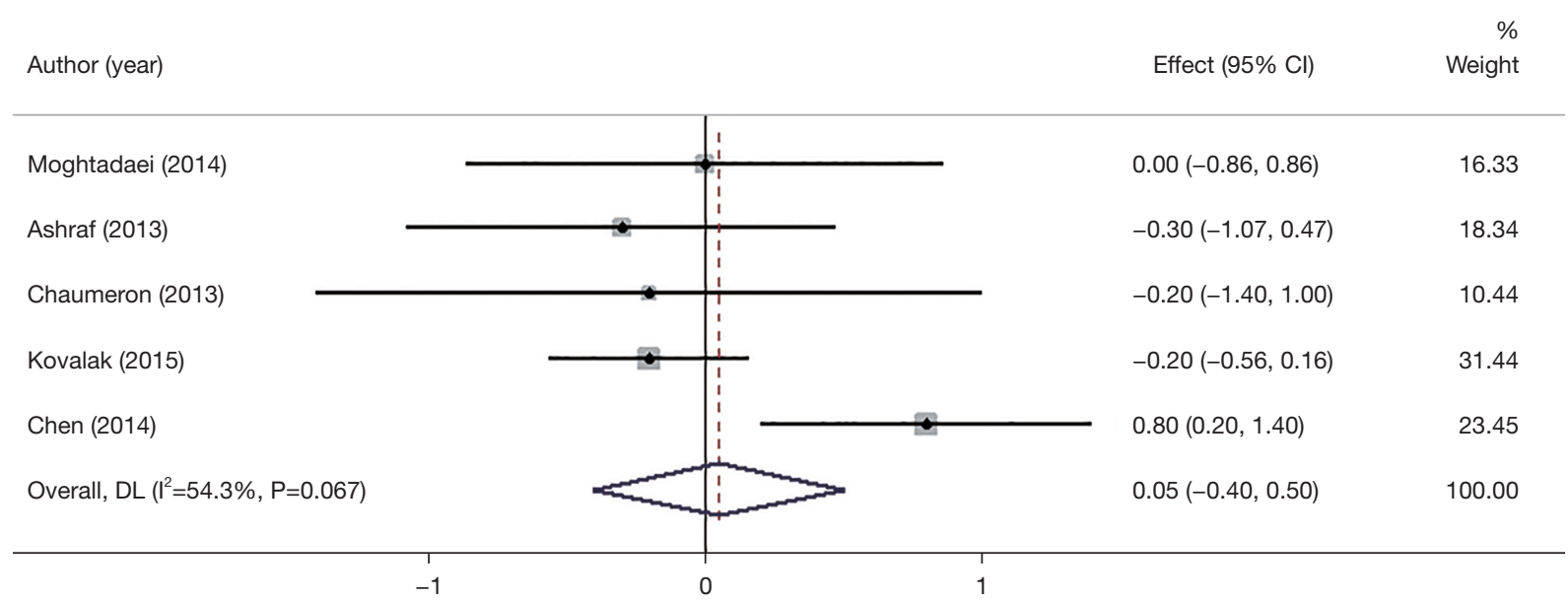

NOTE: Weights are from random-effects model

Figure 6 Forest plot of hospital stay. Comparison of hospital stay between the LIA and FNB anesthesia groups. Statistical method: inverse variance of the random effects model (MD and 95\% CI). LIA, local infiltration anesthesia; FNB, femoral nerve block; MD, mean difference; CI, confidence interval.

As one of the current multi-modal analgesic methods, the introduction of LIA technology into joint replacement surgery was first reported in 2008 (22), which involved the application of a mixture of various analgesics (known as a cocktail) during surgery or before sutures intra-articular soft tissue injection. The main injection sites include the posterior capsule of the knee joint, the collateral ligament, the joint capsule incision, the quadriceps, and the subcutaneous soft tissue. This technology eliminates the stimulation and conduction of pain caused by surgical trauma to achieve the purpose of preventing and relieving postoperative pain, and has the advantage of providing a clear analgesic effect.

Ideal postoperative analgesia should not only provide good pain control and minimal adverse reactions, but also allow patients to move the knee joint as early as possible, and should also be easy to operate. Compared with FNB, LIA does not suffer from motor block and muscle 
strength decline during the rehabilitation period, which is cheaper for patients, and faster and more convenient for clinicians $(16,18)$. At the same time, LIA does not affect the quadriceps muscle strength of the adductor tube block. A previous meta-analysis showed that LIA could significantly improve the postoperative pain score and opioid consumption (23). The results of this study also showed that the effect of LIA is similar to that of FNB, but it can reduce the use of opioids. In summary, LIA, which is effective and safe and feasible for analgesia, may be a more suitable choice for clinicians in pain control after total

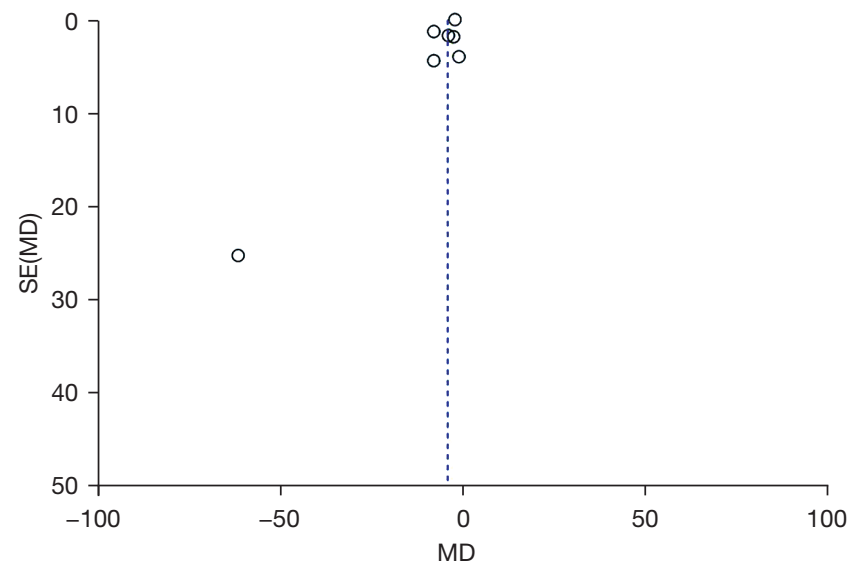

Figure 7 Funnel plot analysis of the possible publication bias in the subgroup Of the amount of opioids used in the $24 \mathrm{~h}$ postoperatively. RR, relative risk; $\mathrm{MD}$, mean difference; $\mathrm{SE}$, standard error of the mean. knee replacement. It is also worth noting that the unskilled operation of the doctor may affect the pain relief effect, and it is necessary to impose practice.

However, the study has some limitations that should be considered. Firstly, the number of included articles was small, and the number of patient cases was also small, which may bias the results of the meta-analysis. Therefore, in the future, multi-center, large-sample studies are needed. Secondly, because this study included fewer outcome indicators, this led to incomplete analysis of many functional rehabilitation results. Third, the influence of publication bias on the results of the analysis should also be considered. Finally, although local anesthetics, nonsteroidal anti-inflammatory drugs, and epinephrine are the most commonly used combinations in peri-articular infiltration as a mixture, the included analysis article reports that the dosage is different, which is worthy of further study. In addition to LIA and FNB, patient-controlled intravenous analgesia and epidural analgesia should also be included in the analysis in future studies.

\section{Conclusions}

In summary, the results of this meta-analysis suggest that LIA and FNB are equally effective in relieving pain, and both are suitable for analgesia after total knee replacement, but LIA can reduce the use of analgesic drugs. Owing to its ease of operation, LIA can be recommended as the priority analgesia for patients with total knee replacement. However, the conclusions of this study are limited by the

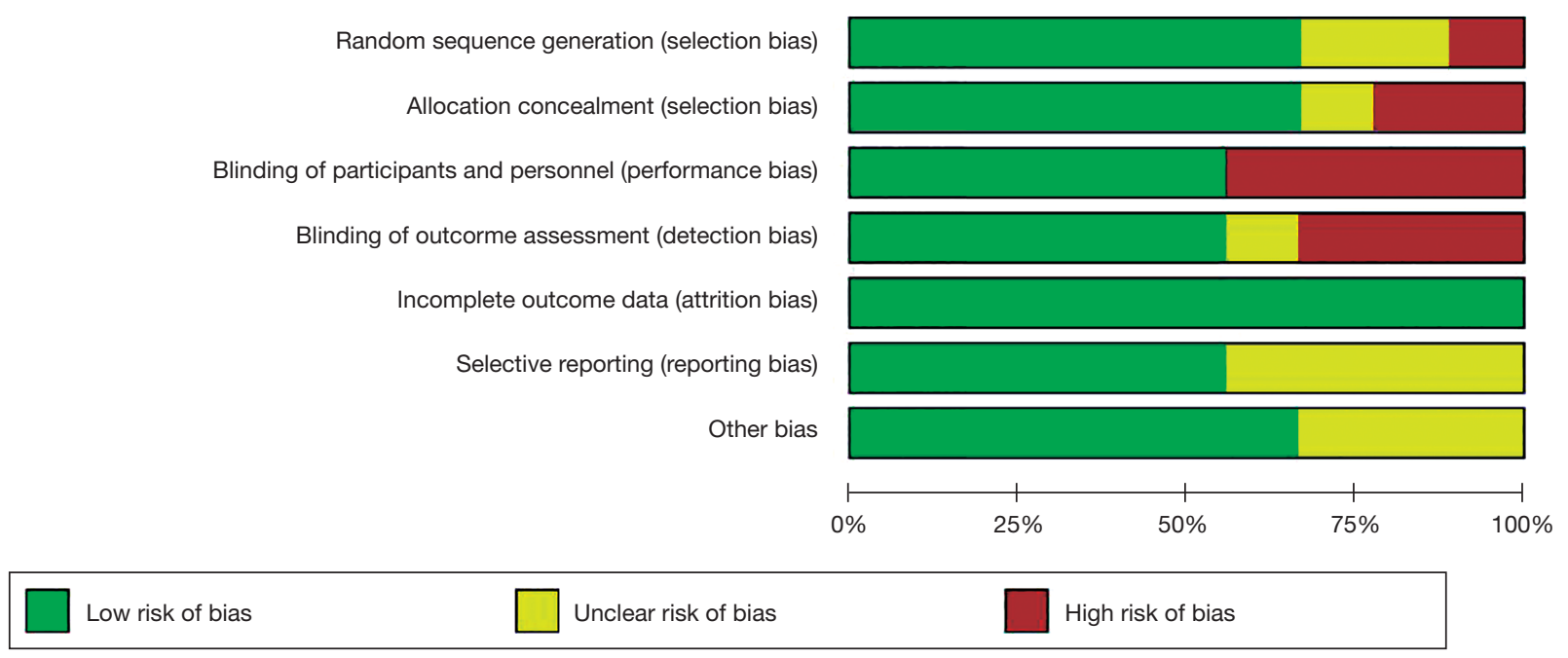

Figure 8 The intensity and distribution of the quality risk of the articles included in the study. 
number and quality of the included studies, and thus, still need to be verified by multi-center, large-sample, high quality randomized controlled trials.

\section{Acknowledgments}

Funding: None.

\section{Footnote}

Reporting Checklist: The authors have completed the PRISMA reporting checklist. Available at https://atm. amegroups.com/article/view/10.21037/atm-22-286/rc

Conflicts of Interest: All authors have completed the ICMJE uniform disclosure form (available at https://atm. amegroups.com/article/view/10.21037/atm-22-286/coif). The authors have no conflicts of interest to declare.

Etbical Statement: The authors are accountable for all aspects of the work in ensuring that questions related to the accuracy or integrity of any part of the work are appropriately investigated and resolved.

Open Access Statement: This is an Open Access article distributed in accordance with the Creative Commons Attribution-NonCommercial-NoDerivs 4.0 International License (CC BY-NC-ND 4.0), which permits the noncommercial replication and distribution of the article with the strict proviso that no changes or edits are made and the original work is properly cited (including links to both the formal publication through the relevant DOI and the license). See: https://creativecommons.org/licenses/by-nc-nd/4.0/.

\section{References}

1. Li S, Luo X, Wang P, et al. Clinical Outcomes of Gap Balancing vs Measured Resection in Total Knee Arthroplasty: A Systematic Review and Meta-Analysis Involving 2259 Subjects. J Arthroplasty 2018;33:2684-93.

2. Ahmed GO, ELSweify K, Ahmed AF. Usability of the AAOS Appropriate Use Criteria (AUC) for the surgical management of knee osteoarthritis in clinical practice. Knee Surg Sports Traumatol Arthrosc 2020;28:2077-81.

3. Davies AF, Segar EP, Murdoch J, et al. Epidural infusion or combined femoral and sciatic nerve blocks as perioperative analgesia for knee arthroplasty. $\mathrm{Br} \mathrm{J}$ Anaesth 2004;93:368-74.
4. Tierney E, Lewis G, Hurtig JB, et al. Femoral nerve block with bupivacaine 0.25 per cent for postoperative analgesia after open knee surgery. Can J Anaesth 1987;34:455-8.

5. Andersen LØ, Kehlet H. Analgesic efficacy of local infiltration analgesia in hip and knee arthroplasty: a systematic review. Br J Anaesth 2014;113:360-74.

6. Andersen LØ, Husted H, Otte KS, et al. A compression bandage improves local infiltration analgesia in total knee arthroplasty. Acta Orthop 2008;79:806-11.

7. Mahadevan D, Walter RP, Minto G, et al. Combined femoral and sciatic nerve block vs combined femoral and periarticular infiltration in total knee arthroplasty: a randomized controlled trial. J Arthroplasty 2012;27:1806-11.

8. McNamee DA, Parks L, Milligan KR. Post-operative analgesia following total knee replacement: an evaluation of the addition of an obturator nerve block to combined femoral and sciatic nerve block. Acta Anaesthesiol Scand 2002;46:95-9.

9. Affas F, Nygårds EB, Stiller CO, et al. Pain control after total knee arthroplasty: a randomized trial comparing local infiltration anesthesia and continuous femoral block. Acta Orthop 2011;82:441-7.

10. Kovalak E, Doğan AT, Üzümcügil O, et al. A comparison of continuous femoral nerve block and periarticular local infiltration analgesia in the management of early period pain developing after total knee arthroplasty. Acta Orthop Traumatol Turc 2015;49:260-6.

11. Ashraf A, Raut VV, Canty SJ, et al. Pain control after primary total knee replacement. A prospective randomised controlled trial of local infiltration versus single shot femoral nerve block. Knee 2013;20:324-7.

12. Chaumeron A, Audy D, Drolet P, et al. Periarticular injection in knee arthroplasty improves quadriceps function. Clin Orthop Relat Res 2013;471:2284-95.

13. Chen D, Jiang J. Comparison of the effects of femoral nerve block and local infiltration anesthesia on pain management after total knee arthroplasty. Chinese Journal of General Practice 2014;12:1867-8.

14. Choi S, O'Hare T, Gollish J, et al. Optimizing Pain and Rehabilitation After Knee Arthroplasty: A Two-Center, Randomized Trial. Anesth Analg 2016;123:1316-24.

15. Kurosaka K, Tsukada S, Seino D, et al. Local Infiltration Analgesia Versus Continuous Femoral Nerve Block in Pain Relief After Total Knee Arthroplasty: A Randomized Controlled Trial. J Arthroplasty 2016;31:913-7.

16. Moghtadaei M, Farahini H, Faiz SH, et al. Pain Management for Total Knee Arthroplasty: Single-Injection 
Femoral Nerve Block versus Local Infiltration Analgesia. Iran Red Crescent Med J 2014;16:e13247.

17. Varshney RK, Prasad MK, Garg M. Comparison of continuous femoral nerve block with local infiltration for postoperative analgesia in unilateral total knee arthroplasty - a randomized controlled trial. Anaesth Pain \& Intensive Care 2019;23:268-73.

18. Kuang MJ, Xu LY, Ma JX, et al. Adductor canal block versus continuous femoral nerve block in primary total knee arthroplasty: A meta-analysis. Int J Surg 2016;31:17-24.

19. Hunt KJ, Bourne MH, Mariani EM. Single-injection femoral and sciatic nerve blocks for pain control after total knee arthroplasty. J Arthroplasty 2009;24:533-8.

20. Krishna Prasad GV. Post-operative analgesia techniques after total knee arthroplasty: A narrative review. Saudi J Anaesth 2020;14:85-90.

Cite this article as: Liu Q, Wang A, Zhang J. The effects of local infiltration anesthesia and femoral nerve block analgesia after total knee arthroplasty: a systematic review and metaanalysis. Ann Transl Med 2022;10(4):178. doi: 10.21037/atm22-286
21. Seet E, Leong WL, Yeo AS, et al. Effectiveness of 3-in1 continuous femoral block of differing concentrations compared to patient controlled intravenous morphine for post total knee arthroplasty analgesia and knee rehabilitation. Anaesth Intensive Care 2006;34:25-30.

22. Kerr DR, Kohan L. Local infiltration analgesia: a technique for the control of acute postoperative pain following knee and hip surgery: a case study of 325 patients. Acta Orthop 2008;79:174-83.

23. Sardana V, Burzynski JM, Scuderi GR. Adductor Canal Block or Local Infiltrate Analgesia for Pain Control After Total Knee Arthroplasty? A Systematic Review and MetaAnalysis of Randomized Controlled Trials. J Arthroplasty 2019;34:183-9.

(English Language Editor: A. Kassem) 